\title{
La gestión de la cadena de valor en un entorno competitivo y cambiante* $^{*}$
}

\author{
The management of the value chain in a competitive \\ and changing environment
}

\section{A gestão da cadeia de valor em um ambiente competitivo e cambiante}

\begin{abstract}
Recibido: 23 de agosto de 2018 Revisado: 17 de septiembre de 2018 Aceptado: 15 de octubre de 2018

Jaime Apolinar Martínew Arroyo*

Universidad Michoacana de San Nicolás de Hidalgo, México

Marco Alberto Valenzo Jiméne: ${ }^{* * *}$

Universidad Michoacana de San Nicolás de Hidalgo, México

Angélica Guadalupe Zamudio De La Cru*****

Universidad Michoacana de San Nicolás de Hidalgo, México

Citar como: Martínez Arroyo, J. A., Valenzo Jiménez, M. A., y Zamudio De La Cruz, A. G. (2019). La gestión de la cadena de valor en un entorno competitivo y cambiante. SIGNOS - Investigación en sistemas de gestión, 11(1), 55-70. DOI: https://doi.org/10.15332/s2145-1389.2019.0001.03

Artículo de resultado de investigación.

* Doctor en Ciencias en Negocios Internacionales, magíster en Administración, especialista en Mercadotecnia, licenciado en Administración. Cadena de Valor, Competitividad, Educación Superior, Universidad Michoacana de San Nicolás de Hidalgo, México. Correo electrónico: jmartinez@umich.mx. ORCID: https://orcid.org/0000-0002-9926-4801

*** Doctor en Ciencias en Negocios Internacionales, magíster en Comercio Exterior, especialista en Mercadotecnia, licenciado en Administración. Cadena de Suministro, Competitividad, Universidad Michoacana de San Nicolás de Hidalgo, México. Correo electrónico: mvalenzo@umich.mx. ORCID: https://orcid.org/0000-0001-6155-5948

${ }^{* * * *}$ Doctora en Ciencias, magíster en Administración, contadora pública. Finanzas, Contabilidad Fiscal, vinculación del sector educativo y empresarial. Facultad de Contaduría y Ciencias Administrativas, Universidad Michoacana de San Nicolás de Hidalgo. México. Correo electrónico: angyzamudio@hotmail.com. ORCID: https://orcid.org/0000-0003-225313-1735
\end{abstract}




\section{RESUMEN}

Este artículo de investigación científica tiene el objetivo de medir las actitudes que muestran las empresas exportadoras de aguacate ubicadas en Uruapan, Michoacán, con respecto a las actividades diferenciadoras tales como gestión ambiental, administración de las relaciones y calidad. La competitividad en tiempos actuales surge como un concepto de vital importancia influenciada por la globalización, se buscan estrategias que se traduzcan en ventajas competitivas que permitan a las empresas obtener una buena posición en el mercado global. Este trabajo tiene un diseño descriptivo correlacional, las variables analizadas fueron a través del software SPSS. Los hallazgos principales muestran que los agentes económicos de la cadena de valor dan una mayor importancia a las relaciones con clientes y proveedores que a la calidad y gestión ambiental, actualmente muy importantes en el terreno de la competencia. En este trabajo se realizó una medición de indicadores, dimensiones y variables identificando con claridad el origen de la fuente de ventaja competitiva.

Palabras clave: competitividad, administración de las relaciones, gestión ambiental, calidad.

\section{ABSTRACT}

This scientific research article aims to measure the attitudes shown by avocado exporting companies, located in Uruapan, Michoacán, regarding differentiating activities such as environmental management, relationship management and quality. In present times competitiveness arises as a concept of vital importance influenced by globalization, searching strategies that turn out into competitive advantages that allow companies to obtain a good position in the global market.
This work has a descriptive correlational design, the variables were analyzed through the SPSS software. The main findings shows that the the value chain economic agents give greater importance to relations with customers and suppliers than to quality and environmental management, currently very important in the competition field. In this work, indicators, dimensions and variables were measured, clearly identifying the background of the competitive advantage source.

Keywords: Competitiveness, relations administration, environmental management, quality.

\section{RESUMO}

Este artigo de pesquisa cientifica tem o objetivo de medir as atitudes que mostram as empresas exportadoras de abacate localizadas em Uruapan, Michoacán, com respeito às atividades diferenciadoras como gestão ambiental, administração das relações e qualidade. A competitividade em tempos atuais surge como um conceito de vital importância influenciada pela globalização, buscam-se estratégias que representem vantagens competitivas que permitam às empresar obter uma boa posição no mercado global. Este trabalho tem um desenho descritivo correlacional, as variáveis analisadas foram a través do software SPSS. As descobertas principais mostram que os agentes econômicos da cadeia de valor dão uma maior importância às relações com clientes e provedores que a qualidade e gestão ambiental, atualmente muito importantes no âmbito da concorrência. Neste trabalho realizou-se uma medição de indicadores, dimensões e variáveis, identificando com clareza a origem da fonte da vantagem competitiva.

Palavras-chave: competitividade, administração das relaciones, gestão ambiental, qualidade. 


\section{INTRODUCCIÓN}

La competitividad surge como uno de los principales conceptos debido a la globalización, pone a todos países en un enlace, con una relación continua. Se desarrollan estrategias nacionales para permitir que los países productores tengan una posición preferencial en el mercado global y manteniendo una continuidad en caso de su efectividad (Bilgen y Varoglu, 2016). Desarrollar estrategias sostenidas para incrementar su prosperidad es la presión de la competitividad global, por lo tanto, las estrategias necesitan los resultados de la investigación científica para determinar con claridad los temas significativos para el análisis de la competitividad. En esta investigación y a pesar de la literatura existente sobre el tema, se propuso un método de medición con el fin de contribuir cualitativamente en el análisis de las fuentes de la competitividad y ventajas competitivas. Esta metodología mide indicadores, dimensiones y variables; la determinación del modelo fue con base en la revisión de la literatura y se utilizó el análisis de la cadena de valor de Porter.

El ambiente de los negocios globales es competitivo, demandante y hostil, para poner a prueba la estrategia competitiva de las organizaciones, afectando y disminuyendo cada ventaja competitiva de la empresa por la presión de la competencia, modificando los mercados a un estado de constante movimiento e inestabilidad, por lo tanto, el ambiente actual de los negocios se halla remotamente estable y predecible. Como resultado, el propósito de esta investigación es identificar fuentes de ventajas competitivas en las actividades de los agentes de la cadena de valor de las empresas exportadoras de aguacate, que les permita competir más eficientemente en los mercados internacionales, especialmente en el mercado norteamericano ante la creciente competencia internacional y nacional.

El siglo XXI ha tenido acontecimientos que son indicadores claros de turbulencia, retos y oportunidades.
La sobrevivencia y éxito en estos tiempos turbulentos dependen de los activos y procesos dentro de una organización que son fuentes de ventajas competitivas, estas fuentes pueden ser tangibles e intangibles (Ambastha y Momaya, 2004). Los activos son los recursos naturales o los recursos creados como infraestructura; los procesos consisten en la transformación de estos activos para lograr ganancias económicas de las ventas a los clientes (DC, 2001).

\section{REVISIÓN DE LITERATURA}

En los mercados internacionales cada país trata de generar ventajas competitivas en varios sectores para mejorar la competitividad internacional de sus productos y expandir sus mercados (Zhang y London, 2013). Habría que decir también que la investigación y especulación sobre la competitividad es extensa e intensiva, la comunidad científica expresa su preocupación por la influencia potencial de esta discusión sobre la aceptación de las decisiones gubernamentales y corporativas a gran escala, que involucra no solo a la industria y al mercado sino también a otras esferas principales de la sociedad. En virtud del hecho de que esta discusión es notable por su esencial naturaleza aleatoria conceptual y terminológica, es necesario abrir una ventana en sus propósitos y proporcionar el término clave en el centro del debate: el concepto de "competitividad".

Este concepto es una categoría sofisticada debido a su aplicación en diversos niveles de generalización, donde toma varios valores y tiene diferentes indicadores de medición. El tema más debatible es una interacción de factores para determinar la dinámica de las relaciones de competencia, con esto en mente y como resultado de una manera natural, se avanza una opinión en donde es imposible especificar cómo es la competitividad: ¿̨es aditiva, lineal o no lineal? Con referencia a cualquier 
objeto, sujeto o proceso, cambia según los propósitos, objetivos y contenidos de estudios de investigación; se revela desde diferentes perspectivas dependiendo del objetivo de la investigación (factores de producción, estrategia, sistema, etc.) y el tema de competitividad (persona, empresas, regiones, país, asociaciones de integración, etc.). En la academia, la literatura sobre competitividad es abundante y se enfatiza en su estudio y análisis, el tema de competitividad es asunto de discusión especialmente en campos de investigación como el comercio internacional, la estrategia económica, la economía global, la geografía económica y la economía aplicada (Chursin y Makarov, 2015).

El Foro Económico Mundial (FEM) define la competitividad como: "El conjunto de instituciones, políticas y factores que determinan el nivel de productividad de un país, y el nivel de productividad, a su vez, establece el nivel de prosperidad que puede alcanzar una economía" (World Economic Forum, 2018). Además, el Instituto Internacional de Gestión y Desarrollo (IMD) afirma que la competitividad de una economía no puede reducirse solo al producto interno bruto (PIB) y a la productividad, porque las empresas también deben hacer frente a los problemas políticos, sociales y dimensiones culturales; por lo tanto, las naciones necesitan proporcionar un entorno que tenga la estructura, las instituciones y las políticas más eficientes que fomenten la competitividad de las empresas (International Institute of Management and Development, 2011).

\section{VENTAJA COMPETITIVA}

Obtener y mantener una ventaja competitiva no parece ser fácil al considerar la complejidad de los mercados locales y extranjeros (Afef Benyoussef Zghidi, 2017). Sin embargo, es importante aclarar que las estrategias basadas en recursos que son raros, valiosos y difíciles de imitar, están demostrando ser más eficientes que otras y están siendo consideradas como los principales motores de la creación de ventajas competitivas sostenibles (Manjeet Kharub, 2017).

Es indudable que la importancia del pensamiento estratégico radica en la obtención de la ventaja competitiva. El propósito de la planeación estratégica es permitir que la empresa obtenga, con la mayor eficacia posible, una ventaja competitiva sobre sus competidores. Tanto es así, que lo que diferencia a la planeación estratégica de todos los demás tipos de planeación en los negocios es, en pocas palabras, la ventaja competitiva (Ohmae, 1983). La ventaja competitiva se define de la siguiente manera: "Cuando una empresa obtiene una mayor tasa de utilidad económica que el promedio de beneficios económicos de otras empresas que compiten en el mismo mercado, la empresa tiene una ventaja competitiva en ese mercado" (Besanko, Dranove y Shanley, 2000).

Una ventaja competitiva sostenida se obtiene cuando una empresa implementa una estrategia de creación de valor que no ha sido simultáneamente implementada por los competidores y estos no son capaces de duplicar los beneficios de esta estrategia (Barney, 1991).

Entonces, la empresa con la ventaja competitiva persigue una estrategia que no ha sido ejecutada por una 0 varias empresas rivales. La estrategia implementada por la organización con ventaja competitiva le proporciona a esta la oportunidad de reducir costos o implementar una estrategia de diferenciación (Porter, 1985).

Es indiscutible que existe una amplia literatura sobre la ventaja competitiva y que ha sido implementada para lograr el éxito de las empresas, y el precursor principal de estas estrategias es el constante incremento de competidores en el ambiente de negocios. De tal manera que, al revisar el uso de la ventaja competitiva en la literatura de la estrategia, el tema común es la creación de valor (Rumelt, 2003). Lo dicho hasta aquí 
supone que la ventaja competitiva puede ser creada de numerosas maneras, por ejemplo, por tamaño de empresa, localización, acceso a los recursos, etc. (Ghemawat, 1986). Las ventajas duraderas vienen del uso del conocimiento para apoyar lo que la empresa hace mejor y agregar valor a los recursos que posee esta y que no están disponibles para los competidores (Goel, Rana y Rastogi, 2010).

\section{GESTIÓN AMBIENTAL}

La globalización hoy en día ha creado un ambiente competitivo mundial en el cual las empresas y los Gobiernos se encuentran altamente involucrados. Por lo tanto, las decisiones estratégicas que se tomen dentro de las organizaciones y los Gobiernos resultan vitales para la mejora de los niveles de competitividad. Actualmente, tanto los países como las empresas están haciendo frente al incremento e intensificación de la competencia mundial, a los rápidos avances tecnológicos, a las nuevas formas de competir y a la cada vez más demandante expectación de los clientes. Por lo tanto, hoy las organizaciones buscan la forma de mejorar su competitividad, las empresas examinan cómo diferenciarse de las demás mediante el desarrollo de actividades especiales o con el uso de recursos propios que les proporcione ventajas competitivas; es importante mencionar que para efectos prácticos de este artículo se manejará el concepto de ventaja competitiva como una forma de ser competitivo, aclarando que tanto la ventaja competitiva como la competitividad son términos diferentes, pero al mismo tiempo son dependientes, ya que el primero representa el medio para ser competitivo y el segundo es el fin u objetivo de la empresa 0 país.

Según la teoría de la economía ambiental, esta interpreta la degradación ambiental como "un fallo del mercado" que se produce cuando el sistema de precios no refleja el deterioro del medio ambiente, o lo que es lo mismo, la escasez de recursos o la contaminación que la producción de un bien genera (Dasgupta, 1990). Es indudable que los temas relacionados con el cuidado del medio ambiente son de alta preocupación para la humanidad (MIT Sloan Management Review, 2009). De acuerdo con la comunidad internacional, en la gestión ambiental se destacan algunas actividades prioritarias tales como: el cambio climático y otras cuestiones, como la calidad y disponibilidad del agua, el aire, la contaminación, la deforestación y los cambios de uso de la tierra, la biodiversidad y la sostenibilidad de la agricultura y la pesca, es decir, en la administración y conservación de los recursos naturales todo estos temas han ganado importancia en la agenda pública debido a su importancia (Yale Center for Environmental Law y Policy; Columbia University, 2010).

Ante esta problemática, recientemente en algunos países se empezaron a observar con interés las consecuencias de las actividades de las empresas en la sociedad y en especial en el medio ambiente, llamando la atención de los medios de comunicación y de algunos sectores de la sociedad. Como resultado la gestión ambiental ha emergido como una prioridad ineludible. Un número creciente de empresas están adoptando sistemas de gestión ambiental como una forma de enfrentar los retos del entorno natural. Muchas de estas también deciden tener sus sistemas de gestión ambiental certificados de acuerdo a una 0 ambas normas internacionales disponibles, ISO 14001 y EMAS (Eco Management de la Unión Europea y auditoría medioambientales). Tanto el sistema de gestión ambiental como el proceso de certificación implican una importante inversión de recursos financieros y de gestión del esfuerzo, lo cual plantea la cuestión de los beneficios que las empresas podrían obtener de estas actividades (Chassagnon y Haned, 2012).

La gestión ambiental, de acuerdo con Prado Román, Blanco González y Díez Martín (2011), es: 
El proceso de identificar un conjunto de estrategias ambientales y de los procesos de la empresa estableciendo un buen alineamiento de ambos. Siendo la planificación un instrumento apropiado para desarrollar adecuadamente un sistema ya que implica un considerable aumento de la complejidad de la gestión y la necesaria coordinación de los procesos y políticas.

Como resultado de la creciente presión de las organizaciones no gubernamentales, autoridades locales, nacionales e internacionales, los consumidores, competidores y otras partes interesadas, la responsabilidad ambiental de una empresa ha adquirido una importancia sin precedentes. Esta tendencia ha provocado que un número creciente de empresas introduzca la dimensión del cuidado al medio ambiente a su estrategia competitiva. La gestión ambiental es ya una disciplina bien establecida dentro de la actividad de la administración. Un sistema de gestión ambiental consiste típicamente en diferentes objetivos y normas para el desempeño ambiental, los procedimientos para el control y la ejecución, y una asignación formal de responsabilidades entre los empleados y sus funciones (Kumar Singh y Ramalinga, 2008). Cabe señalar que regularmente las empresas compiten a través de una estrategia basada en el precio y la calidad, sin embargo, cada vez son más las organizaciones que compiten en temas ambientales y por medio de estos poder obtener una ventaja competitiva.

Es importante mencionar que por varias razones los problemas ambientales que enfrenta la sociedad no pueden ser resueltos sin la participación de los intereses empresariales (Hoffman, 2005). Por lo tanto, las empresas están cada vez más preocupadas por la importante influencia que las prácticas ambientales tienen sobre su competitividad (Walley y Whitehead, 1994). De hecho, aquellos que no son conscientes de la ventaja competitiva que puede ser derivada de las prácticas ambientales, no están dispuestos a invertir en tecnologías de cuidado al medio ambiente sino hasta después de haber conocido los límites de la legislación.
En algunos casos, las empresas desarrollan recursos únicos, valiosos e inimitables, recursos que son derivados de sus prácticas medioambientales (Hart, 1995). Estas actividades son las que permiten a las empresas lograr una ventaja competitiva con un producto diferenciado, al mismo tiempo que mejora las relaciones con instituciones reguladoras, con las comunidades locales y con grupos ambientales (Christmann, 2000). Es decir, estas prácticas permiten a las empresas lograr una ventaja competitiva basada en el cuidado al medio ambiente. Por otro lado, gran parte de la literatura sugiere que el entrenamiento de la gerencia y de los empleados sobre el cuidado del medio ambiente y de la consciencia, son algunas de las acciones que, junto con grandes dosis de motivación, hacen posible que las empresas al mismo tiempo mejoren su desempeño ambiental y varias dimensiones de la competitividad (Alberti, Cain, Calabrese y Rossi, 2000).

En este mismo sentido, empresas líderes de opinión han citado que la gestión ambiental produce un beneficio inmediato en la imagen de la empresa, haciendo hincapié en que la creación de valor así como la reducción de costos son recompensas, las cuales pueden convertirse en fuente de ventaja competitiva (MIT Sloan Management Review, 2009). Es importante también mencionar que esta fórmula varía según el país debido a los muchos factores involucrados.

\section{CALIDAD}

La calidad es un proceso que requiere que una compañía tenga tenacidad y experiencia con este proceso, además esta estrategia permite que una compañía mejore sus mediciones de las utilidades y el nivel de defectos. E indudablemente, el desempeño de una compañía puede ser mejorado cuando todos los empleados están involucrados a través de la administración de la calidad total. 
La calidad es definida como: "el enfoque en la satisfacción de las necesidades del cliente", lo que conduce a un proveedor a trabajar para dar al cliente un producto que cumpla o supere las expectativas (Sila y Ebrahimpour, 2003). Aunque no existe una definición universalmente aceptada para la administración de la calidad total, el proceso recae en los teóricos para desarrollar un tema común que sea visto universalmente en programas de calidad; programas que contengan los temas comunes utilizados por la mayoría de las empresas como: la mejora continua, el enfoque al cliente, la administración de recursos humanos y la administración de procesos. Otros autores como Shiba, Graham y Walden (1995), definen la calidad mediante el estudio de las diferentes facetas por las cuales ha pasado el concepto de calidad a lo largo de la historia: a) aptitud según estándares, b) aptitud de uso, c) aptitud de costos, d) aptitud para necesidades latentes, e) aptitud hacia la cultura corporativa y, f) aptitud con el entorno social y global. El concepto de calidad abarca, según Dochy (1988), los siguientes campos: satisfacción de los clientes, satisfacción de las necesidades de los trabajadores y satisfacción de las necesidades de la sociedad.

La administración de la calidad total (ACT) es una dirección estratégica que muchas empresas escogen u optan por llevarla a través de los mercados competitivos a los que se enfrentan hoy en dí. La alta dirección debe entender dos áreas importantes cuando implementa un programa de calidad total: los objetivos de la organización y los cambios en las iniciativas de la organización. El proceso de implantación de un programa de calidad total involucra a todos los empleados, desde el presidente de la compañía hasta los funcionarios de limpieza y todos los proveedores y clientes externos, este compromiso incluye la mejora continua y la manufactura esbelta, reingeniería y seis sigma ${ }^{1}$

1 Seis sigma (o six sigma) es una metodología de mejora de procesos creada en Motorola por el ingeniero Bill Smith en la década de los 80, esta metodología está centrada en la reducción de la
(Soltani, Lai y Gharneh, 2005). Por lo tanto, un sistema de gestión de calidad es necesario para que una empresa fabrique un producto de calidad que cumpla o supere los requerimientos del cliente (Elshennawy, 2004). Deming (1982) describe que el principal objetivo de la administración de la calidad total es desarrollar y mantener una ventaja competitiva a través de una eficiencia mayor. Esta eficiencia es manifestada en la reducción de los costos y en la mejora de la satisfacción del cliente. Simultáneamente, algunos estudios han demostrado que la ACT puede generar una ventaja competitiva basada en los costos o en la diferenciación (Reed, Lemak y Montgomery, 1996), la cual algunas veces es sostenida.

A su vez, evidencia proveniente de Powell (1995) indica que las empresas que adoptaron la ACT obtuvieron una ventaja competitiva sobre las empresas que no la adoptaron. En este mismo sentido, Chang (2005) expresa que la administración del sistema de calidad puede ser el vehículo de la mejora continua de una organización y así poder mantener la ventaja competitiva en el mercado.

\section{ADMINISTRACIÓN DE LAS RELACIONES}

Actualmente varias empresas tienen el reto de enfrentar el mercado de una manera distinta, en el que el punto medular es el enfoque al cliente. Bajo este contexto tan especial, la línea que divide al éxito del fracaso de las propuestas empresariales es muy delgada, ya que está supeditada a la lealtad del cliente.

variabilidad, consiguiendo reducir o eliminar los defectos o fallos en la entrega de un producto o servicio al cliente (tomado de http:// www.leansolutions.co/conceptos/que-es-six-sigma/). 
Por lo mismo, para cumplir este cometido, es muy importante establecer y mantener relaciones de largo plazo que se transformen en lealtad y que incrementen las transacciones de negocios a través del tiempo entre los clientes y la organización. Así, el objetivo estratégico debe ser generar fidelidad del consumidor hacia la compañía (Greenberg, 2003), al considerase o definirse al siglo XXI como la era de las relaciones en las que los propósitos principales son la creación de confianza, comunicación recíproca, compromiso y respeto mutuo (Olasz, 2006). Los clientes más influyentes toman sus decisiones de compra con base en las relaciones que mantienen con sus proveedores, de tal forma que las empresas mantienen en sus clientes a su principal activo y su objetivo prioritario es establecer, desarrollar y mantener el negocio a partir de los clientes que proporcionan un mayor valor, así como los de mayor crecimiento, para lograr una rentabilidad superior.

El ambiente cambiante, especialmente la crisis energética, el progreso en la industria de servicios y concentrarse en la administración de la calidad, obligan a las compañías a cambiar su enfoque sobre la adquisición de clientes, al enfoque de retención de clientes (Sheth, 2002), por medio de la construcción de relaciones con estos y agregar más valor a los productos y servicios (Lindgreen y Wynstra, 2005). Por otro lado, es muy claro que el impacto en el negocio por no adoptar la interacción con los clientes se transforma en la insatisfacción de ellos y la pérdida de oportunidades de ingresos. Las compañías pueden adoptan la administración de relaciones con los clientes (ARC) para obtener una ventaja distinta sobre los competidores. Esto requiere proveer herramientas e información a sus clientes; hoy en día la ARC da una ventaja competitiva a las empresas de tal forma que se convierte en un imperativo para mantener las expectaciones del mercado (Sedgewick, 2004). De manera similar, las relaciones con los clientes son vistas como importantes recursos que se ajustan a cuatro criterios: son valiosos, raros, inimitables e insustituibles. Peppers y Rogers (2005) mencionan que las compañías deben tratar a los clientes como recursos escasos, sin enfocarse excesivamente en el corto plazo. Desde la perspectiva de la teoría basada en los recursos, los clientes y las relaciones con los clientes son vistos como la base para obtener una ventaja competitiva (Gouthier y Schmid, 2003).

La compañía que conoce mejor a los clientes que la competencia, tiene una gran oportunidad de incrementar su participación en el mercado. Enseñar a la empresa más y más acerca de las necesidades y preferencias de los clientes, provee a esta con una inmensa ventaja competitiva. Por lo tanto, más clientes buscan específicamente a la compañía porque les provee lo que quieren y como lo quieren, lo que resulta más difícil de copiar para la competencia (Pine, Peppers y Rogers, 1995). De igual manera, la ventaja de toda la información colectada por la empresa mediante la aplicación de la administración de las relaciones con los clientes, es vista como una ventaja competitiva de la empresa.

\section{PROBLEMÁTICA}

El entorno competitivo se ha complicado poco a poco para los exportadores de aguacate michoacanos, tanto en el plano nacional como en el internacional.

En marzo de 2015, La Comisión Federal de Competencia Económica (COFECE) emitió una resolución que dio oportunidad de exportar aguacate hass a EE. UU. a los empacadores de todo el territorio nacional, terminando con el monopolio que tenía la Asociación de Productores y Empacadores de Aguacate, denominada APEAM. Esta resolución tiene como objetivo suprimir y corregir la práctica monopólica relativa a la venta atada -conducta en la que un proveedor condiciona la venta de un producto, bien 
o servicio, a comprar o contratar otro distinto-, con lo que imposibilitaba la exportación de aguacate hass a EE. UU. a todos aquellos empacadores que no estuvieran afiliados a este último organismo.

La COFECE detalló que la APEAM es el único organismo (cooperador) autorizado para la administración, facturación y cobranza de los servicios de supervisión y verificación fitosanitaria del Departamento de Agricultura de Estados Unidos (USDA-APHIS), los cuales son necesarios para poder exportar aguacate hass de México a ese país.

Señaló entonces la Comisión Federal de Competencia Económica (COFECE) que:

Esta asociación condicionaba la prestación de sus servicios de administración, facturación y cobranza de los servicios de supervisión y verificación fitosanitaria de USDA-APHIS a que: 1) los empacadores necesariamente se afiliaran a la APEAM y pagaran una cuota de acceso y, 2) al pago y/o contratación de servicios adicionales, no necesarios para exportar aguacate Hass a EU en términos del Plan de Trabajo signado entre las autoridades mexicanas y de EU, tales como el desarrollo de proyectos de investigación, implementación de campañas de promoción en el mercado de EU, monitoreo de mercado en EU, cabildeo ante autoridades de agricultura de dicho país, contratación de un seguro de responsabilidad para proteger a los empacadores, utilización del Sistema Integral de Información de Cosecha (SICOA) y realizar proyectos de apoyo a la comunidad (Info-Hass, 2015).

A partir de esta resolución, la producción de aguacate en México se ha incrementado principalmente en los estados de Jalisco, México, Guerrero y Chiapas, aumentándose el número de hectáreas cultivadas con aguacate, como se observa en Tabla 1.
Tabla 1. Hectáreas plantadas de aguacate en estados mexicanos

\begin{tabular}{|l|c|c|}
\hline \multicolumn{1}{|c|}{ Estado } & $\begin{array}{c}\text { Total de hectáreas } \\
\text { cultivadas }\end{array}$ & Incremento (\%) \\
\hline Michoacán & 159.328 & 0.7 \\
\hline Jalisco & 21.022 & 7.6 \\
\hline Estado de México & 9.946 & 12 \\
\hline Guerrero & 4.439 & 0.4 \\
\hline Chiapas & 3.264 & 8.5 \\
\hline
\end{tabular}

Fuente: Secretaría de Agricultura, Ganadería, Desarrollo Rural, Pesca y Alimentación - Servicios de Información Agroalimentaria y Pesquera (SAGARPA-SIAP).

Las exportaciones de aguacate de Jalisco antes del último fin de semana del Super Bowl (o Supetazón) alcanzaron un valor de 220 millones de dólares; esto no hubiera sido posible sin el dictamen de la COFECE. Es evidente en este caso la competencia directa a los exportadores michoacanos.

En el plano internacional, otro aspecto que se evalúa es el número de toneladas por hectárea. La producción de México, y en especial de los aguacateros michoacanos, está entre regular y baja, pero si se compara con el mejor país productor la diferencia en muy amplia; esto se observa en la Tabla 2. en Latinoamérica varios países han incrementado sus exportaciones al mercado norteamericano, como son Colombia, República Dominicana y Perú, los cuales se unen a competidores ya establecidos como California y Chile (Info-Hass, 2015). Ver Tabla 2.

Tabla 2. Producción en toneladas por hectárea

\begin{tabular}{|l|c|}
\hline País & T/ha \\
\hline Samoa & 29.8 \\
\hline Marruecos & 26.3 \\
\hline Rep. Dominicana & 25.9 \\
\hline Perú & 10.8 \\
\hline México & 10.1 \\
\hline Chile & 4.3 \\
\hline
\end{tabular}

Fuente: Organización de las Naciones Unidas para la Alimentación y la Agricultura (FAO) 2018. 
Lo mencionado anteriormente se refleja en las exportaciones mexicanas de aguacate a diversos países del mundo, que se han visto disminuidas en el último ciclo como se muestra en la Tabla 3.

Tabla 3. Volumen de exportaciones mexicanas de aguacate

\begin{tabular}{|l|r|l|c|}
\hline \multicolumn{2}{|c|}{ MY julio 2015-junio 2016 } & \multicolumn{2}{c|}{ MY julio 2016-junio 2017 } \\
\hline EE. UU. & 742.971 & EE. UU. & 668.095 \\
\hline Japón & 63.944 & Japón & 61.716 \\
\hline Canadá & 62.148 & Canadá & 71.607 \\
\hline Otros & 91.299 & Otros & 72.245 \\
\hline Total & 960.342 & Total & 873.963 \\
\hline
\end{tabular}

Fuente: Global Trade Information Services, Inc. Global Trade Atlas, México Edition, junio 2017.

Desde luego que este panorama de una mayor competencia para los exportadores michoacanos los obliga a mejorar en varios aspectos buscando ser más competitivos, por lo tanto, la pregunta general de investigación es:

¿Cuáles son las variables que incrementan la competitividad de las empresas de la cadena de valor del aguacate ubicadas en Uruapan, Michoacán?

\section{METODOLOGÍA}

El presente artículo emana de una investigación científica y tiene un diseño descriptivo-correlacional, ya que describe al objeto de estudio, además, determina la correlación que tiene la variable independiente con la variable dependiente y las ventajas competitivas en la cadena de valor de las empresas exportadoras de aguacate ubicadas en Uruapan, Michoacán, México.

Primeramente, se estableció contacto con el gerente de la Comisión Michoacana del Aguacate para determinar con exactitud la problemática observada y realizar el mapeo de la cadena de valor del aguacate. Luego se recopiló información del universo de cada eslabón de la cadena de valor: viveristas, productores, empacadores y transportistas. Posteriormente se elaboró un cuestionario compuesto por 46 preguntas respecto a las variables observadas: calidad, administración de las relaciones y gestión ambiental, y que contiene una escala tipo Likert de cinco rangos. Se realizó una prueba piloto y para efectos de esta investigación se aplicaron 16 cuestionarios entre los diferentes agentes de la cadena de valor del aguacate, en los cuales las personas encuestadas realizaron algunas observaciones con relación a la redacción y cantidad de las preguntas, así como también acerca del lenguaje técnico de las variables utilizadas. Por lo tanto, se procedió a solucionar las observaciones hechas por los encuestados. La aplicación de esta prueba permitió también medir la confiabilidad del cuestionario utilizando el software SPSS 22, resultando el alfa de Cronbach de 0.914. Por lo que resulta ser un cuestionario con consistencia interna para ser aplicado en la recopilación de la información.

\section{MODELO DE VARIABLES}

La Figura 1 muestra el modelo de variables utilizado en esta investigación, así como las dimensiones e indicadores.

\section{RESULTADOS Y DISCUSIÓN}

Aspectos vitales posteriores a la elaboración del cuestionario son la medición de la validez y la confiabilidad. La validez de un cuestionario es un aspecto de la misma importancia que la confiabilidad, la validez está relacionada con la revisión del marco teórico. Una buena revisión contribuye a que el diseño del cuestionario sea válido (Rivas, 2004) y la confiabilidad con la consistencia interna del cuestionario, es decir, que produce resultados similares en diversas ocasiones, la Tabla 4 muestra los resultados de confiabilidad. 
Figura 1. Modelo de variables

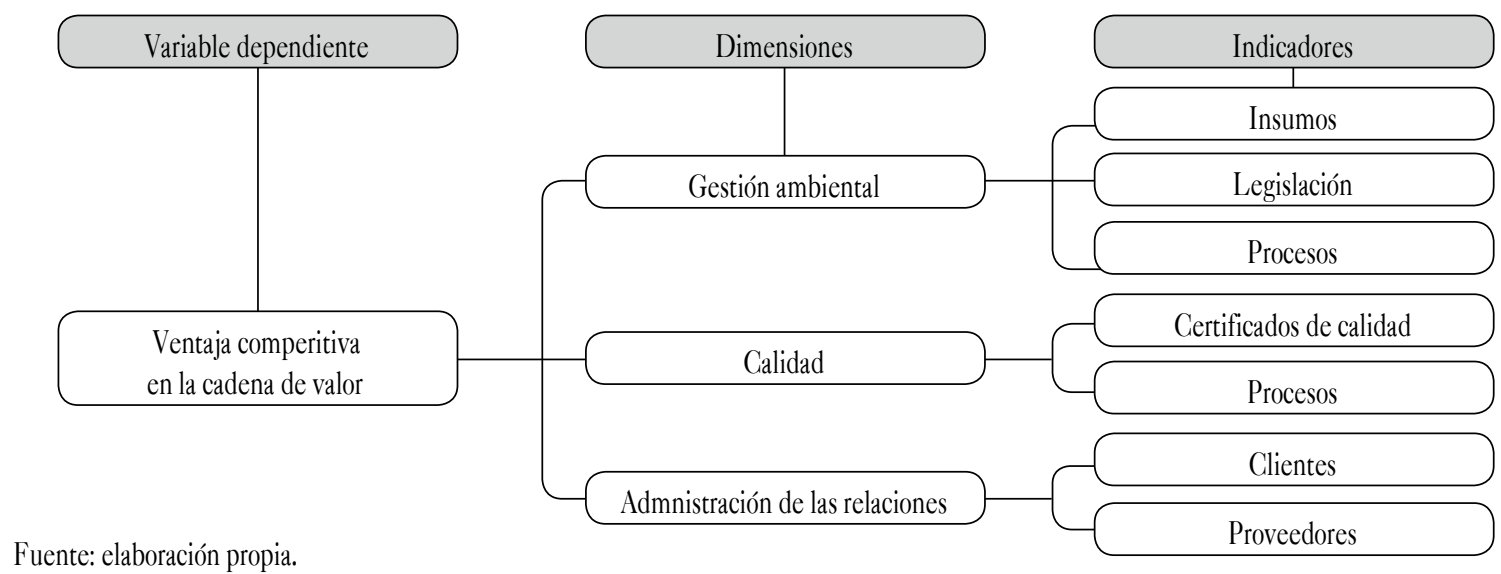

Tabla 4. Análisis de confiabilidad

\begin{tabular}{|c|c|c|c|c|}
\hline Elemento & Cuestionario & Gestión ambiental & Calidad & Administración de las relaciones \\
\hline alfa de Cronbach & 0.962 & 0.942 & 0.922 & 0.968 \\
\hline
\end{tabular}

Fuente: elaboración propia.

\section{CORRELACIÓN DE SPEARMAN}

Figura 2. Coeficiente de correlación de Spearman y determinación

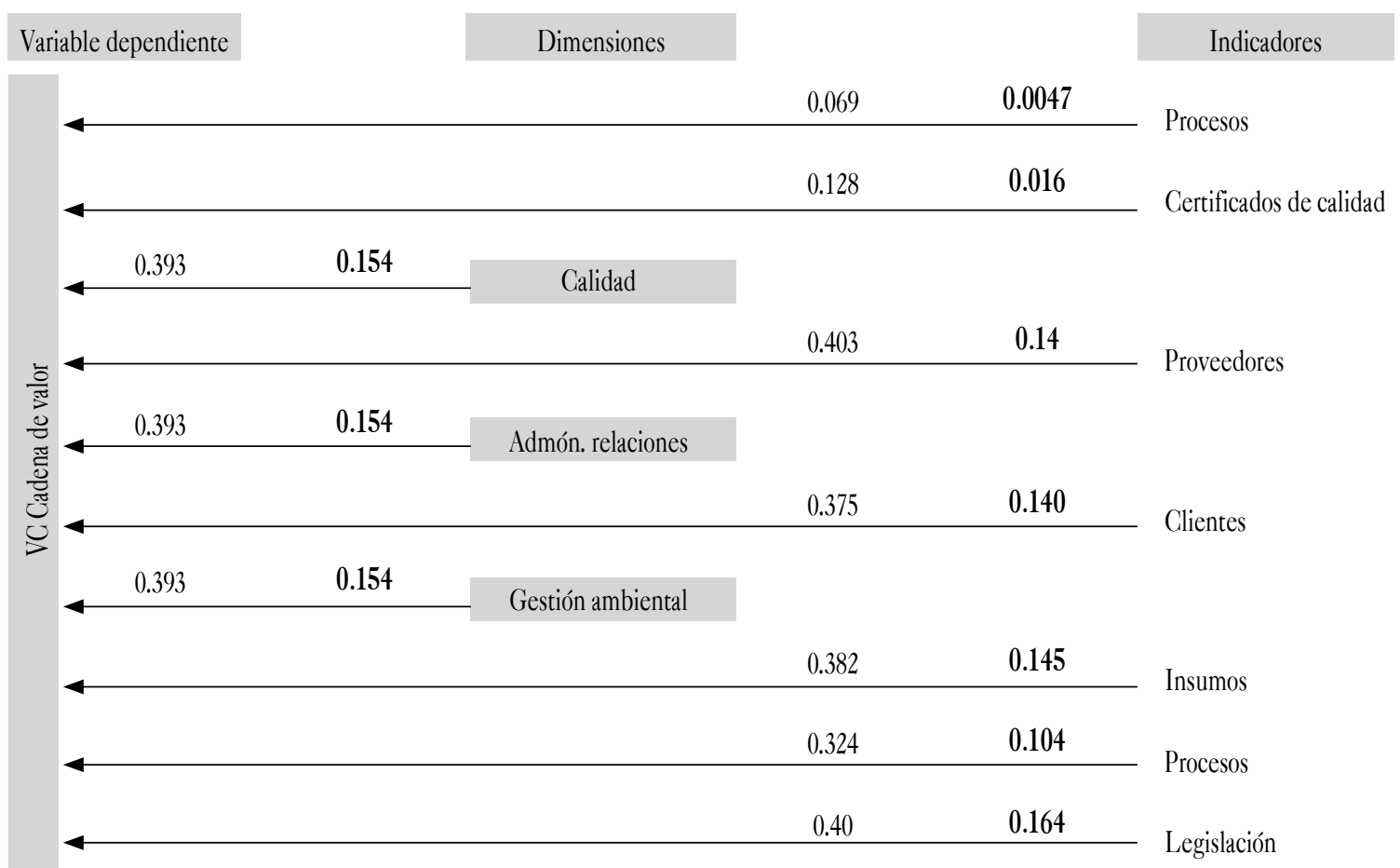

Fuente: elaboración propia. 
El coeficiente de correlación de Spearman, al igual que el de Pearson, muestra una asociación entre variables. A diferencia del anterior, permite obtener un coeficiente de asociación entre variables que no se comportan normalmente, entre variables ordinales. Se calcula con base en una serie de rangos asignados; la Figura 2 muestra los resultados de correlación.

\section{RESULTADOS GENERALES}

Los resultados generales de las dimensiones analizadas como fuentes de competitividad por agente económico en relación con su desempeño dentro de la cadena de valor del aguacate, se muestran en la Tabla 5.

Los resultados ponen en evidencia que viveristas y productores de aguacate tienen un bajo rendimiento en las dimensiones evaluadas, sin embargo, los empacadores muestran una mejor eficiencia, sin duda alguna esto representa un desbalance en la cadena de valor ya que la esencia de toda cadena es generar el mayor nivel de valor para trasladarlo al siguiente eslabón y que al final los productos sean de alto valor agregado.

\section{VERIFICACIÓN DE HIPÓTESIS}

En este apartado se presentan las pruebas que permiten contrastar si dos muestras aleatorias e independientes proceden de una misma población, es decir, si un factor que subdivide la población de origen incide de forma significativa sobre el valor central de la población. Estos contrastes son alternativas no paramétricas al análisis de la varianza cuando se incumple alguno de los supuestos básicos de dicho análisis. El único requisito para aplicar estos contrastes es que la variable esté medida al menos en una escala ordinal. La prueba de

Tabla 5. Resultados generales de la muestra por dimensión

\begin{tabular}{|l|l|l|l|l|l|}
\cline { 2 - 6 } \multicolumn{1}{c|}{} & Muy bajo & Bajo & Regular & Alto & Muy alto \\
\hline Gestión ambiental & & & & & \\
\hline Viveristas & & & & & \\
\hline Productores & & & & & \\
\hline Empacadores & & & & & \\
\hline Calidad & & & & & \\
\hline Viveristas & & & & & \\
\hline Productores & & & & & \\
\hline Empacadores & & & & & \\
\hline Admón. relaciones & & & & & \\
\hline Viveristas & & & & & \\
\hline Productores & & & & & \\
\hline Empacadores & & & & \\
\hline
\end{tabular}

Fuente: elaboración propia 
hipótesis dio como resultado retener la hipótesis nula en el análisis de las dimensiones: gestión ambiental y calidad; por el contrario, se rechaza la hipótesis nula en la dimensión administración de las relaciones. Por lo tanto, la variable gestión ambiental muestra que su desempeño no es importante para estas empresas ya que dan poca importancia al cuidado del medio ambiente cuando efectúan sus actividades permanentemente. De hecho, existe un constante y creciente cambio del uso de suelo, las plantaciones de pino benéficas para el medio ambiente se están talando de manera irracional para plantar árboles de aguacate, estos últimos altamente nocivos para el medio ambiente, no toman en cuenta la legislación ambiental en sus procesos e insumos y no averiguan si sus proveedores tienen implementado un sistema de gestión ambiental. Con respecto a la variable calidad, se presenta la misma situación, y en lo referente a la administración de relaciones, estas organizaciones sí desempeñan acciones destinadas a mantener unas relaciones a largo plazo ya que de esto depende mucho su supervivencia y permanencia en el mercado norteamericano.

\section{CONCLUSIONES}

Esta investigación se justifica por la necesidad de las empresas exportadoras de aguacate mexicanas de encontrar fuentes de ventaja competitiva, ya sea temporal o sostenida, para mantener su cuota en el mercado norteamericano. Las barreras de entrada a las exportaciones de otros países al mercado norteamericano existentes han desaparecido por el dictamen de La Comisión Federal de Competencia Económica, por lo tanto, todo productor de aguacate nacional puede exportar a EE. UU., siempre y cuando cumpla con los requisitos establecidos por la USDA, con esto, las empresas michoacanas pierden una importante ventaja competitiva que las obliga a mejorar para competir por este importante mercado.

La competencia por el mercado norteamericano de aguacate se ha incrementado paulatinamente en los últimos años, más países latinoamericanos han entrado a la producción y exportación de ese producto agrícola; por ejemplo, República Dominicana con un

Tabla 6. Prueba de hipótesis

\begin{tabular}{|l|l|c|l|}
\hline \multicolumn{1}{|c|}{ Variable } & \multicolumn{1}{|c|}{ Test } & \multicolumn{1}{c|}{ Siǵnificancia } & \multicolumn{1}{c|}{ Decisión } \\
\hline Gestión ambiental & $\begin{array}{l}\text { Independent-Samples } \\
\text { Kruskal-Wallis Test }\end{array}$ & .314 & Retener la hipótesis nula \\
\hline Calidad & $\begin{array}{l}\text { Independent-Samples } \\
\text { Kruskal-Wallis Test }\end{array}$ & .179 & Retener la hipótesis nula \\
\hline Administración de las relaciones & $\begin{array}{l}\text { Independent-Samples } \\
\text { Kruskal-Wallis Test }\end{array}$ & .000 & Rechazar la hipótesis nula \\
\hline \multicolumn{2}{|r|}{ Significación asintótica se muestra. El nivel de significancia es de .05. } \\
\hline
\end{tabular}

Fuente: datos de la investigación. 
rendimiento por hectárea que casi triplica a los productores de Michoacán. En este mismo sentido, Colombia también ha incrementado su rendimiento y su volumen de exportación, Perú es otro competidor con una tasa de crecimiento constante en la producción y exportación de aguacate. En el plano nacional, los productores del estado de Jalisco han aumentado la cosecha de aguacate con el incremento en la superficie plantada, complementada con la apertura para comercializar su fruto a los Estados Unidos. En este año 2018 exportó 22 mil toneladas de aguacate para los festejos del Super Bowl realizado en enero del presente año. También varios estados de México han entrado al cultivo de aguacate y en algún momento se iniciará la cosecha. Australia, Nueva Zelanda, Kenia, España, Suráfrica, Chile, el estado de California, Florida e Israel, son productores en crecimiento constante y representan una seria amenaza a los productores michoacanos de aguacate, ya que la mayoría apunta a exportar al mercado norteamericano el cual tiene o muestra un medio no satisfecho del $22 \%$. Los productores michoacanos han incrementado la superficie plantada originando problemas por el cambio en el uso del suelo, ya que han deforestado una gran superficie de plantaciones de pino ocasionando afectaciones al medio ambiente.

Las organizaciones aguacateras enfrentan una competencia masiva interna y externa y compiten también por una mejor posición en el mercado y por sobrevivir, emplean técnicas conocidas, las rutinas son consagradas por el tiempo con hábitos basados en la experiencia. La toma de decisiones viene de los procesos sobre la base de las experiencias pasadas que da el trabajo, la confianza y el instinto. Los resultados de esta investigación muestran que los agentes de la cadena no realizan actividades clave para ser mejores competidores, tales como la calidad y la gestión ambiental, actualmente tan necesarias para la imagen y prestigio de las compañías que las realizan.

\section{REFERENCIAS}

Afef Benyoussef Zghidi, I. Z. (2017). Service orientation as a strategic marketing tool: The moderating effect of business sector. Competitiveness Review: An International Business Journal, 27(1), 40-61. DOI: 10.1108/CR-02-2015-0012.

Alberti, M., Cain, M., Calabrese, A., y Rossi, D. (2000). Evaluation of the costs and benefits of an environmental management system. International Journal of Production Research, 38(17), 55-66.

Ambastha, M., y Momaya, K. (2004). Competitiveness of firm: Review of theory, frameworks, and models. Singapore Management Review, 1(26), 45-61.

Barney, J. B. (1991). Firm resources and sustained competitive advantage. Journal of Management, 17, 99-120.

Besanko, D., Dranove, D., y Shanley, M. (2000). Economics of strategy. New York: Wiley \& Sons.

Bilgen, H. I., y Varoglu, A. (2016). Methodology research of competitiveness and simple application for turkey's defense industry. Competitiveness Review, 26(5), 537-558. DOI:10.1108/ CR-10-2015-0081.

Chassagnon, V., y Haned, N. (2012). Environmental innovation and innovation leadership: A firm-level empirical analysis. Lyon, Francia: ESDES Business School.

Christmann, P. (2000). Effects of the "best practices" of environmental management on cost advantage the role of complementary assetts. Academy of $\mathrm{Ma}$ nagement Journal, 4(43), 663-80.

Chursin, A., y Makarov, Y. (2015). Management of competitiveness theory and practice. Cham, 
Suiza: Springer International Publishing AG. DOI: 10.1007/978-3-319-16244-7.

Dasgupta, P. S. (1990). The environment as a commodity. Oxford Review of Economic Policy, 6, 51-67.

DC. (Octubre de 2001). Destination competitiveness. Development of a Model with Application to Australia and the Republic of Korea. Australia: An Australian Goyt Report.

Deming, W. (1982). Quality, productivity, and competitive position center for advanced engineering studies. Cambridge, MA: Massachusetts Institute of Technology.

Elshennawy, A. (2004). Quality in the new age and the body of knowledge for quality engineers. Total Quality Management, 15(5-6), 603-614.

Ghemawat, P. (1986). Sustainable advantage. Harvard Business Revierw, 5(64), 53-58.

Goel, A., Rana, G., y Rastogi, R. (2010). Knowledge management as a process to develop sustainable competitive advantage. South Asian Journal of $\mathrm{Ma}$ nagement, 17(3), 104.

Gouthier, M., y Schmid, S. (2003). Customers and customer relationships in service firms: The perspective of the resource based view. Marketing Theory, $3(1), 119-143$.

Greenberg, P. (2003). Las claves del CRM. Gestión de las relaciones con los clientes. (I. D. España, ed.) Madrid, España: McGraw-Hill.

Hart, S. (1995). A natural resource-based view of the firm. Academy of Management Review, 20(4), 986-1014.

Hoffman, A. (2005). Business decisions and the environment: significance, challenges, and momentum of an emerging research field', in social and behavioral science research priorities for environmental decision making. Washington D. C.: Environmental Protection Agency and the National Science Foundation.

Info-Hass. (24 de marzo de 2015). Info-Hass. Net. Recuperado de http://www.infohass.net/mercadousa

International Institute of Management and Development. (2011). Methodology and Principles of Analysis. Lausana, Suiza: IMD.

Kumar Singh, R., y Ramalinga, M. H. (2008). Development and implementation of environmental strategies for steel industry. Int. J. Environmental Technology and Management, 8(1), 70-72.

Lindgreen, A., y Wynstra, F. (2005). Value in business markets: What do we know? Where are we going? Industrial Marketing Management, 34(7), 732-48.

Manjeet Kharub, R. S. (2017). Comparative analyses of competitive advantage using Porter diamond model (The ase of Msmes in Himachal Pradesh). Competitiveness Review: An International Business Journal, 27(2), 132-160. DOI: 10.1108/ CR-02-2016-0007.

MIT Sloan Management Review. (2009). Sustainability and competitive advantage. Boston, MA: Sloan Review. MIT. Edu.

Ohmae, K. (1983). La mente del estratega. México D. F., México: McGraw-Hill.

Olasz, C. (2006). Marketing's role in a relationship age. Baylor Business Review, 24(2).

Páramo Morales, D. (Junio de 2001). Hacia la construcción de un modelo de cultura organizacional orientada al mercado. Revista Colombiana de Marketing, 2(6), 1-26. 
Jaime Apolinar Martínez Arroyo, Marco Alberto Valenzo Jiménez, Angélica Guadalupe Zamudio De La Cruz

Peppers, D., y Rogers, M. (2005). Return on customer. Creating maximum value from your scarcest resource. Marshall Cavendish Business.

Pine, B. J., Peppers, D., y Rogers, M. (1995). Do you want to keep your customers forever? Harvard Business Review, 73(2), 103-114.

Porter, M. (1985). Competitive advantage (vigésima reimpresión, ed.). (F. Press, ed.) México D. C., México: Editorial Continental.

Prado Román, C., Blanco Gonzaléz, A., y Díez Martín, F. (2011). Hacia un nuevo modelo de gestión medioambiental. Observatorio Medioambiental, 14, 69-91.

Reed, R., Lemak, D., y Montgomery, J. (1996). Beyond process: TQM content and firm performance. Academy Of Management Reviewe, 21(1), 173-202.

Rivas, T. L. (2004). Cómo hacer una tesis de maestría. (IPN, ed.) México D. F., México: Taller Abierto.

Rumelt, R.P. (2003). What in the world is competitive advantage? Policy Working Paper, 5.

Sedgewick, R. (2004). Real-time CRM: A competitive advantage today, a competitive imperative tomorrow?CustomerInter@ction Solutions, 22(8), 50.

Shiba, S., Graham, A. y Wladen, D. (1995). TQM desarrollos avanzados: cuatro revoluciones en el pensamiento sobre dirección. Madrid, España: TGP Hoshin.
Sheth, J. (2002). The future of relationship marketing. Journal of Services Marketing, 16(7), 590-2.

Sila, I., y Ebrahimpour, M. (2003). Examination and comparison of the critical factors of total quality management (TQM) across countries. International Journal of Production Research, 41(2), 235-268.

Soltani, E., Lai, P., y Gharneh, N. (2005). Breaking through barriers to TQM effectiveness: Lack of commitment of upper-level management. Total Quality Management, 8-9, 1009-1021.

Walley, N., y Whitehead, B. (1994). 'It's not easy being green. Harvard Business Revierw, (72), 46-52.

World Economic Forum. (2018). The Global Competitiveness Report. Génova, Suiza: WEF.

Yale Center for Environmental Law y Policy; Columbia University. (2010). 2010 Environmental Performance Index. Columbia: World Economic Forum.

Zhang, P., y London, K. (2013). Towards an internationalized sustainable industrial competitiveness model. Competitiveness Revierw, 23(2), 95-113. DOI: 10.1108/10595421311305325 